\title{
Maren Elisabeth Schwab, Antike begreifen: Antiquarische Texte und Praktiken in Rom von Francesco Petrarca bis Bartolomeo Marliano (Quellen und Untersuchungen zur Lateinischen Philologie des Mittelalters, Band 22)
}

\author{
Stuttgart: Anton Hiersemann Verlag, 2019, XI, 471 S. mit 34 Abb., ISBN \\ 978-3-7772-1908-0, €168.
}

\section{Ingo Herklotz ${ }^{1}$}

Accepted: 18 February 2021 / Published online: 20 March 2021

(c) The Author(s) 2021

Das Thema des Antiquarianismus hat in den letzten Jahren eine kaum noch überschaubare Flut von Forschungen hervorgebracht. M. E. Schwab richtet ihren Blick auf ,das lange 15. Jahrhundert“ in Rom (welches Jahrhundert wäre nicht in jüngerer Zeit als ,lang“" etikettiert worden?), das dem Titel zufolge von Petrarca bis zu Bartolomeo Marliani reicht, wie die Lektüre erweist, aber schon mit den Mirabilia urbis Romae, also bald nach 1140, einsetzt, denn dieser vielfach bearbeitete Text hat noch Gelehrte wie Paolino da Venezia und Flavio Biondo nachdrücklich geprägt. Irritierend liest sich die einleitende Behauptung der Autorin, die hier ihre latinistische Dissertation vorlegt und somit aus der Textforschung kommt, die vorangehenden Studien zu den Antiquaren hätten sich vornehmlich mit literarischen Hervorbringungen, weniger mit Objekten befasst. Als gäbe es nicht seit mehr als hundert Jahren Untersuchungen zur Grabungs- und zur Sammlungsgeschichte (zu dieser über die von Schwab zitierte Literatur hinaus auch A. Cavallaro, $\mathrm{Hg}$., Collezioni di antichità a Roma fra ' 400 e '500, Rom 2017) und als wisse man nicht seit geraumer Zeit, dass die Erforschung antiker Sachkultur das Rückgrat gerade der als mores et instituta bekannten antiquarischen Teildisziplin ausmachte, welch letztere während des 16. und 17. Jahrhunderts innerhalb der Altertumswissenschaft die führende Methode verkörperte. Ähnliche Fehleinschätzungen des Forschungsstandes sind auch auf anderen Seiten des Buches festzustellen, obwohl der Autorin die einschlägige Fachliteratur in der Regel durchaus bekannt geworden ist. Antiquare sind für sie dann „Personen, die mit großem Eifer die Begegnung mit antiken Artefakten suchen“ (S. 13). Diese Artefakte laufen bei Schwab - in bewusster Verengung des Sprachgebrauchs von Antike und Renaissance - unter der Bezeichnung antiquitates. Die

Ingo Herklotz

ingo.herklotz@uni-marburg.de

1 Kunstgeschichtliches Institut, Philipps-Universität, Biegenstraße 11, 35037 Marburg, Germany 
Erforschung „materialer antiquitates“ steht im Mittelpunkt des Buches, das sich somit zum material turn bekennt, wobei die Autorin in nahezu jedem Kapitel vor Augen führt, dass eine Beschäftigung mit den Objekten keineswegs aus sich selbst erfolgen konnte, sondern stets der Kurzschließung mit Inschriften und literarischen Quellen Trennung bitte korrigieren: bedurfte. Vor eben diesem Hintergrund hatte der Rezensent den Antiquarianismus von seinen Erkenntnisinteressen und nicht von den Methoden her definiert, nämlich als eine Sparte der Altertumswissenschaft, die sich mit all jenen Aspekten antiker Zivilisation auseinandersetzte, die nicht oder nicht primär die histoire événementielle betrafen, dabei materielle Zeugnisse der Vergangenheit auswerten konnte, aber nicht zwangsläufig musste. Aufschlussreich mag es in diesem Zusammenhang erscheinen, wenn der erfolgreichste antiquarische Autor des 16. Jahrhunderts, Johannes Rosfeld, nie in Italien gewesen ist.

Berechtigt wirkt Schwabs Einwand, dass der Antiquarianismus heute Gefahr läuft, zu sehr als Vorgeschichte der neueren Disziplinen verstanden zu werden. Ausgeblendet bleibe dabei ein ,imaginative antiquarianism“ (Angus Vine), welcher der Auseinandersetzung mit dem Altertum in der Renaissance durchaus eignete. Wie sehr gelehrte Anstrengungen und Phantasie einander durchdringen konnten, Trennung bitte korrigieren: belegt die Autorin mit Werken wie Petrarcas Africa-Epos und der Hypnerotomachia Polyphili des Francesco Colonna (1499), deren gewinnbringende Analyse ihr Buch wie ein roter Faden durchzieht. Ihre Betrachtung macht die originellsten Abschnitte der hier angezeigten Neuerscheinung aus. Auch dieser eher spielerische Umgang mit der Antike ist freilich schon verfolgt worden, so etwa in dem dreibändigen, von Salvatore Settis herausgegebenen, hier allerdings nicht zitierten Werk La memoria dell'antico, Turin 1984-1986, oder in Leonard Barkans Unearthing the Past, New Haven 1999, der gerade den Dichtungen über antike Skulpturen ein hohes Maß an Aufmerksamkeit zugestand.

Um das Interesse der Altertumswissenschaftler an den Objekten jenseits der Kunstwerke herauszustellen, widmet die Autorin dem berühmten Fund einer angeblich gut erhaltenen Mädchenleiche an der Via Appia (1485) und dem jüngst durch John McManamon (2016) ausführlichst untersuchten Bemühen um die antiken Schiffswracks im See von Nemi jeweils ein eigenes Kapitel (Kap. 3-4). Der mit Ciceros Tochter Tullia identifizierte Frauenkörper erschien den begeisterten Zeitgenossen wie eine ,pagane Heilige“. Allerdings fragt man sich, warum die in Abb. 14 wiedergegebene Zeichnung ihn unbekleidet zeigt. So hatte man den Körper nicht aufgefunden. Wollte man ihn auf diese Weise doch wieder als heidnisch charakterisieren? Alle anderen Kapitel bewegen sich in den gewohnten Bahnen: Sie handeln von Inschriften und Münzen (Kap. 2), von Monumenten und Ruinen (Kap. 5-6) sowie von römischer Topographie (Kap. 6-7), wobei den frühen Stadtplänen besonderes Gewicht zukommt (ergänzend zu diesen M. Bevilacqua und M. Fagiolo, Hg., Piante di Roma dal Rinascimento ai Catasti, Rom 2012). Die Untersuchung fragt dann nach den antiquarischen „Praktiken“ im Umgang mit den Altertümern. Man ging in der Stadt spazieren, um sie zu besichtigen, kopierte und fälschte Inschriften und versuchte, deren epigraphische Ausformungen nachzuahmen, man fertigte Abgüsse von Münzen und begann sogar diese zu wiegen, man dokumentierte die Funde in Zeichnungen und führte Vermessungen von Ruinen aus. Antiquare, deren Einschätzungen auf Autopsie beruhten, faszinieren die Autorin offenbar besonders. 
Immer wieder ging es anschließend darum, die Objekte in Texte umzusetzen, die auf deren „Wiederbelebung“, mithin auch auf eine „christliche Rahmung“, abzielten. Kurzum, hier wird dem Leser noch einmal das gesamte Spektrum antiquarischer Aktivitäten vor Augen geführt. Alte Bekannte, viele von ihnen schon im vierten Band des Codice topografico della città di Roma von Valentini und Zucchetti vereint (1953), begegnen ihm dann auch bei den behandelten Autoren: Manuel Chrysoloras, Poggio Bracciolini, Felice Feliciano, Lorenzo Valla, Alberti, Biondo, Fazio degli Uberti, Giovanni Dondi, Brunelleschi, Ciriaco d'Ancona, Raffael, der Anonymus Magliabecchianus und Bartolomeo Marliani - sie alle gehören zu den Protagonisten dieses Buches. Beachtung verdient die Relativierung des lange Zeit überbewerteten Francesco Petrarca, dessen Beitrag zur römischen Topographie sich als zuhöchst textlastig und zudem ganz unter der Wirkung der Mirabilientradition erweist. (Zu Petrarcas konservativer Sicht der Antike auch P. Seiler, in Pratum Romanum - Richard Krautheimer zum 100. Geburtstag, Wiesbaden 1997, S. 299-324.) Anders als die neuere Forschung hatte Onofrio Panvinio schon im 16. Jahrhundert die Mirabilien am Anfang einer antiquarischen Erforschung Roms gesehen. Mit Blick auf die Wirkungsgeschichte der älteren Texte (Mirabilia und Graphia) gibt Schwab ihm recht, gelangt dann aber doch nur zu einer zwiespältigen Wertung: „Es fällt schwer, diese mittelalterliche Konstruktion des antiken Roms als , antiquarisch ' zu bezeichnen.“ (S. 299) Warum dieser Vorbehalt? Worum es dem Verfasser der Mirabilia bei seinen Gegenüberstellungen ,ubi fuit ... nunc est ... “ ging, war es dann auch weniger, die von Huber-Rebenich und anderen behauptete Kontinuität zwischen antikem und zeitgenössischem Rom aufzuzeigen; vielmehr lag dem Text daran, das heidnisch-antike Rom allenthalben unter, hinter und neben den christlichen Bauten aufzuspüren, und dieses Bemühen ist fraglos antiquarisch. Verfehlt scheint es darüber hinaus, die Statuenlegenden der Textgruppe noch immer im Bereich der SchwankLiteratur zu verorten. Gerade der Abschnitt zur Reiterstatue des Marc Aurel gibt eine politisch durchaus brisante Umbenennung des zuvor mit Konstantin assoziierten und somit kirchlich vereinnahmten Werks zu erkennen und rückt zugleich erstmal die raison d'être einer antiken, insbesondere republikanischen Ehrenstatue mit antiquarischem Verständnis ins Licht. (Dazu V. Wiegartz, Antike Bildwerke im Urteil mittelalterlicher Zeitgenossen, Weimar 2004, S. 116-20.) Um die richtige Benennung des Reiters kreisen im Übrigen noch die Texte des Quattrocento. Es erstaunt, wenn Schwab die vielleicht interessanteste Bearbeitung des Mirabilientextes, die des aus England stammenden Magisters Gregorius (nach 1220), nicht in ihre Diskussion einbezieht. Gerade sie verrät an vielen Stellen eine unmittelbare Begegnung mit der Antike und hätte die Bedeutung des Manuel Chrysoloras im Hinblick auf die Autopsie durchaus relativieren können.

Für manche der hier aufgezeigten Diskurse vermisst man ein wenig das antike Fundament. Zwar ist gelegentlich von Marcus Varro die Rede, viel weniger aber von Publius Victor und den antiken Regionenbeschreibungen, von der Diskussion über die Roma quadrata, die man schon im Altertum führte (dazu A. Grandazzi, in: Mélanges de l'Ecole Française de Rome - Antiquité, 105, 1993/2, S. 493-545) oder von der antiken Ekphrasis, welche die von ihr beschriebenen Protagonisten bereits zum Sprechen brachte. Andererseits hätte sich die von Schwab erzählte Geschichte auch für das 16. Jahrhundert noch fortschreiben lassen. Ein veritables Programm der 
objektfixierten Altertumswissenschaft wurde gerade dort aufgestellt, wo die vorliegende Untersuchung endet, in dem bekannten Vorhaben, das Claudio Tolomei 1542 für die Accademia della Virtù formulierte. In der zweiten Hälfte des Jahrhunderts griffen die Gelehrten des Farnese-Kreises dann einige dieser Anregungen auf.

Der Fachmann wird in Schwabs Darstellung wohl nur Weniges finden, was er so oder ähnlich nicht schon andernorts gelesen hat. Von Kapitel zu Kapitel wechselnd sind die angesprochenen Themen zu unterschiedlich, als dass es zu einem vertieften Eindringen in den jeweiligen Diskurszusammenhang kommen könnte. Für den interessierten Laien entsteht dagegen ein überaus anschaulich geschildertes Bild von der bunten Vielfalt antiquarischer Umtriebe bei Beginn der Neuzeit, das dem aktuellen Stand zumindest der angelsächsischen und der deutschen Forschung weitgehend entspricht. Ein solcher Leser wird dieses Buch mit großem Gewinn zu Rate ziehen. Schade nur, dass der Band so teuer ist.

Funding Open Access funding enabled and organized by Projekt DEAL.

Open Access This article is licensed under a Creative Commons Attribution 4.0 International License, which permits use, sharing, adaptation, distribution and reproduction in any medium or format, as long as you give appropriate credit to the original author(s) and the source, provide a link to the Creative Commons licence, and indicate if changes were made. The images or other third party material in this article are included in the article's Creative Commons licence, unless indicated otherwise in a credit line to the material. If material is not included in the article's Creative Commons licence and your intended use is not permitted by statutory regulation or exceeds the permitted use, you will need to obtain permission directly from the copyright holder. To view a copy of this licence, visit http://creativecommons.org/licen ses/by/4.0/.

Publisher's Note Springer Nature remains neutral with regard to jurisdictional claims in published maps and institutional affiliations. 